\title{
Estimación de biomasa y carbono almacenado en árboles de oyamel afectados por el fuego en el Parque Nacional "El Chico", Hidalgo, México
}

\section{Biomass estimation and carbon stock in fir trees affected by fire in "El Chico" National Park, Hidalgo, México}

\author{
Ramón Razo-Zárate ${ }^{1}$, Alberto J. Gordillo-Martínez¹, \\ Rodrigo Rodríguez-Laguna², C. César Maycotte-Morales ${ }^{2}$ \\ y Otilio A. Acevedo-Sandoval ${ }^{1}$
}

\begin{abstract}
RESUMEN
Se efectuó un estudio en el Parque Nacional El Chico, Hidalgo, con el objetivo de estimar la biomasa y el carbono aéreo almacenado en los árboles muertos en pie, en los árboles adultos vivos y en el renuevo establecido en forma natural de Abies religiosa "oyamel" después de 12 años de ocurrido un incendio forestal de tipo superficial que afectó 30,34 ha. Se realizó un inventario forestal de 15 sitios circulares de $1000 \mathrm{~m}^{2}$ distribuidos de forma sistemática, para medir las variables de diámetro normal y altura. Para determinar la biomasa se utilizó el valor de densidad de la madera de oyamel $\left(360 \mathrm{~kg} / \mathrm{m}^{3}\right)$ y para el coeficiente de carbono se tomaron muestras que fueron analizadas con el equipo Solids TOC Analyzer $\circledast$, obteniendo un valor de 0,45 . Las ecuaciones generadas para estimar biomasa y carbono en árboles de Abies religiosa en un área afectada por un incendio forestal fueron $B=0,06463^{*} D N^{2,38322}$ y $C=0,029083^{*} D N^{2,38322}$ respectivamente, ambas mostraron un buen ajuste $\left(R^{2}=0,99\right)$, por lo que son adecuadas y confiables para usarse en áreas siniestradas con condiciones similares, para la misma especie y región, como Áreas Naturales Protegidas donde no es posible utilizar métodos destructivos. En las 30,34 ha siniestradas por el incendio forestal ocurrido en 1998 en el Parque Nacional El Chico, no se consumieron por el fuego un total de $665,05 \mathrm{t}$ de carbono que no fueron liberadas hacia la atmósfera, sumándose una captura actual de 297,33 t de carbono por el renuevo establecido a 12 años de ocurrido el siniestro.
\end{abstract}

PALABRAS CLAVE:

Abies religiosa, árboles muertos, ecuaciones alométricas, incendio forestal, renuevos.

\begin{abstract}
In "El Chico", National Park, in Hidalgo, México is a study was carried out with the objective of estimating the biomass and carbon stored in the dead trees standing, in the live adult trees and seedlings established in natural form of Abies religiosa "oyamel" after 12 years of a forest fire of superficial type that affected 30,34 hectares. A forest inventory was made on 15 circular sites of $1000 \mathrm{~m}^{2}$ each, distributed in a systematic way, in order to measure the variables of normal diameter and height. To determine the biomass the density value of fir wood was used $\left(360 \mathrm{~kg} / \mathrm{m}^{3}\right)$ and for the carbon coefficient the Solids TOC Analyzer® was utilized, obtaining a value of 0,45 . The generated equations to estimate
\end{abstract}


the biomass and carbon in trees of Abies religiosa in an area affected by a forest fire were $B=0,06463 * D N^{2,38322}$ and $C=0,029083$ * $D N^{2,38322}$ respectively, showing a good adjustment $\left(R^{2}=0,99\right)$; these equations are considered to be suitable and reliable to be used in damaged areas with similar conditions, for the same species and for regions such as Natural Protected Areas where it is not possible to use destructive methods. In 30,34 damaged hectares of the "El Chico" National Park, there were a total of 665,05 tons of stock carbon not consumed by the fire, to which must be added a current capture of 297,33 tons of carbon by the seedling established in the twelve years from the disaster.

KEY WORDS:

Abies religiosa, dead trees, alometric equations, fire forest, seedlings.

\section{INTRODUCCIÓN}

Los bosques y selvas capturan, almacenan y liberan carbono como resultado de los procesos fotosintéticos, de respiración y de degradación de materia seca; son considerados como los ecosistemas terrestres responsables de la mayor parte de los flujos de carbono entre la tierra y la atmósfera (Tipper, 1998). El almacenamiento neto de carbono orgánico en los bosques depende del manejo dado a la cobertura vegetal, edad, distribución de tamaños, estructura y composición de ésta. El servicio ambiental que proveen los bosques y selvas como secuestradores de carbono permite reducir la concentración de este elemento en la atmósfera, misma que se incrementa debido a las emisiones producto de la actividad humana (Torres y Guevara, 2002).

En general es aceptado que la tasa de fijación de carbono por medio de procesos de fotosíntesis es más alta en rodales jóvenes que en rodales maduros, pero el almacenamiento total de carbono en el sistema es mayor en los bosques maduros (Cadena y Ángeles, 2005), considerando que en los ecosistemas terrestres el carbono queda retenido en la biomasa aérea, mantillo, madera muerta, biomasa subterránea y en el suelo a través del tiempo (IPCC, 2000). De esta manera, los ecosistemas de bosques se destacan por su gran capacidad de fijar carbono en sus estructuras, de manera particular lo fijan en forma estable en la parte leñosa. Así, se ha estimado que los árboles asimilan y almacenan grandes cantidades de carbono durante toda su vida (Ordóñez et al., 2001) y en el fuste de un árbol completo es donde se almacena aproximadamente $84 \%$ de biomasa de la cual $46 \%$ es carbono (Avendaño et al., 2009).

El escenario ideal para la fijación y almacenamiento de carbono por los bosques es aquel en el que las masas forestales se mantienen dinámicas mediante la incorporación constante de materia orgánica al suelo proveniente de los árboles adultos, mientras se va estableciendo la regeneración natural de las distintas especies y otros individuos jóvenes están en plena actividad fotosintética. Dicho escenario es susceptible a los efectos nocivos de algunos fenómenos naturales tales como incendios forestales, plagas, enfermedades y presencia de huracanes, cuando ocurren fuera de su régimen histórico o natural o los causados por el uso inadecuado de los bosques y selvas. Uno de los agentes de alteración más significativos son los incendios forestales, que en muchas ocasiones liberan en unos cuantos minutos grandes cantidades del carbono, en forma de bióxido de carbono (Ikkonen et al., 2004), que los bosques almacenaron por muchos años y ocasionan daños al arbolado y sus recursos asociados. Sin embargo, la presencia del fuego en la mayoría de los bosques templados favorece el establecimiento de la regeneración natural.

La estructura, composición florística, diversidad, distribución y extensión geográfica de los bosques de Abies han sido afectadas por los aprovechamientos 
forestales sin control, los incendios forestales frecuentes, la ganadería extensiva, el crecimiento de centros de población y la conversión de zonas boscosas a terrenos de cultivo o pastizales (Cuevas et al., 2011). Por lo anterior, las estrategias para la conservación y el manejo a largo plazo de estos bosques deben reconocer el papel histórico del disturbio causado por el fuego, así como el potencial de cambios en la intensidad de los mismos y sus efectos ecológicos (Fulé y Covington, 1997).

Después de ocurrido un incendio forestal, para la valoración de los daños normalmente se calcula la superficie y el volumen de madera afectada y, si acaso, algunas veces se observa la afectación al paisaje. La mayoría de ocasiones no se evalúa la cantidad de árboles que logran sobrevivir al siniestro, los fustes de los árboles muertos que permanecen por mucho tiempo en el sitio y que se incorporan al suelo, por esto, es necesario evaluar la cantidad de biomasa y carbono residual posterior a un incendio para complementar los informes que se emiten a las dependencias oficiales y para programar actividades de restauración de los sitios afectados. Para conocer lo anterior, existen ecuaciones matemáticas que permiten determinar la biomasa de cada árbol, a partir de variables de fácil medición (diámetro normal, altura total) y a bajo costo; las cuales pueden tener validez local o regional, generada para una especie o grupo de especies (Schroeder et al., 1997).

\section{OBJETIVO}

Estimar la biomasa y el carbono aéreo almacenado en los árboles muertos que permanecen en pie, en aquellos árboles que lograron sobrevivir y el renuevo establecido en forma natural de Abies religiosa, después de 12 años de ocurrido un incendio forestal. Además de comparar las ecuaciones generadas en este estudio con las desarrolladas para la misma especie en un bosque del estado de Tlaxcala.

\section{METODOLOGÍA}

El estudio se realizó dentro del Parque Nacional El Chico que corresponde al primer Parque Nacional decretado en México; se ubica en el extremo occidental de la Sierra de Pachuca entre las coordenadas $20^{\circ} 10^{\prime} 10^{\prime \prime}$ y $20^{\circ} 13^{\prime} 25^{\prime \prime}$ de latitud norte y $98^{\circ} 41^{\prime} 50^{\prime \prime}$ y $98^{\circ} 46^{\prime} 02^{\prime \prime}$ de longitud oeste. Comprende una superficie total de 2739 ha (Conanp, 2005), de las cuales 30,34 ha se afectaron por un incendio forestal de tipo superficial ocurrido en 1998 (año atípico nacional donde ocurrieron incendios de gran magnitud). Esta área se ubica al noroeste del parque entre las coordenadas geográficas $20^{\circ} 11^{\prime} 18^{\prime \prime}$ y $20^{\circ} 12^{\prime} 29^{\prime \prime}$ de latitud norte y $98^{\circ} 42^{\prime} 57^{\prime \prime}$ y $98^{\circ} 44^{\prime} 41^{\prime \prime}$ de longitud oeste (Fig. 1).

Con base en el sistema de Köppen modificado por García (1981), en el Parque Nacional se presenta un clima $\mathrm{C}(\mathrm{m})$ (w) b (i') gw" que corresponde a un templado subhúmedo con lluvias en verano, la temperatura media anual oscila entre $12^{\circ} \mathrm{C}$ y $18{ }^{\circ} \mathrm{C}$, existiendo influencia de monzón y presentándose un porcentaje de lluvia invernal menor de $5 \%$ de la total anual. El verano es fresco y largo, con inviernos fríos con poca oscilación térmica y presencia de canícula.

El tipo de roca predominante es ígnea extrusiva del tipo brecha volcánica y andesita. Los suelos predominantes son del tipo Cambisol húmico, Regosol dístrico y Andosol húmico de textura media. La vegetación en la mayor parte del parque está formada por bosques de oyamel, con distintas condiciones de productividad. Las principales especies arbóreas son Abies religiosa (H.B.K.) Schl. et Cham., Quercus spp. y Pseudotsuga macrolepis Flous (Amezcua y Valderrama, 1999). 


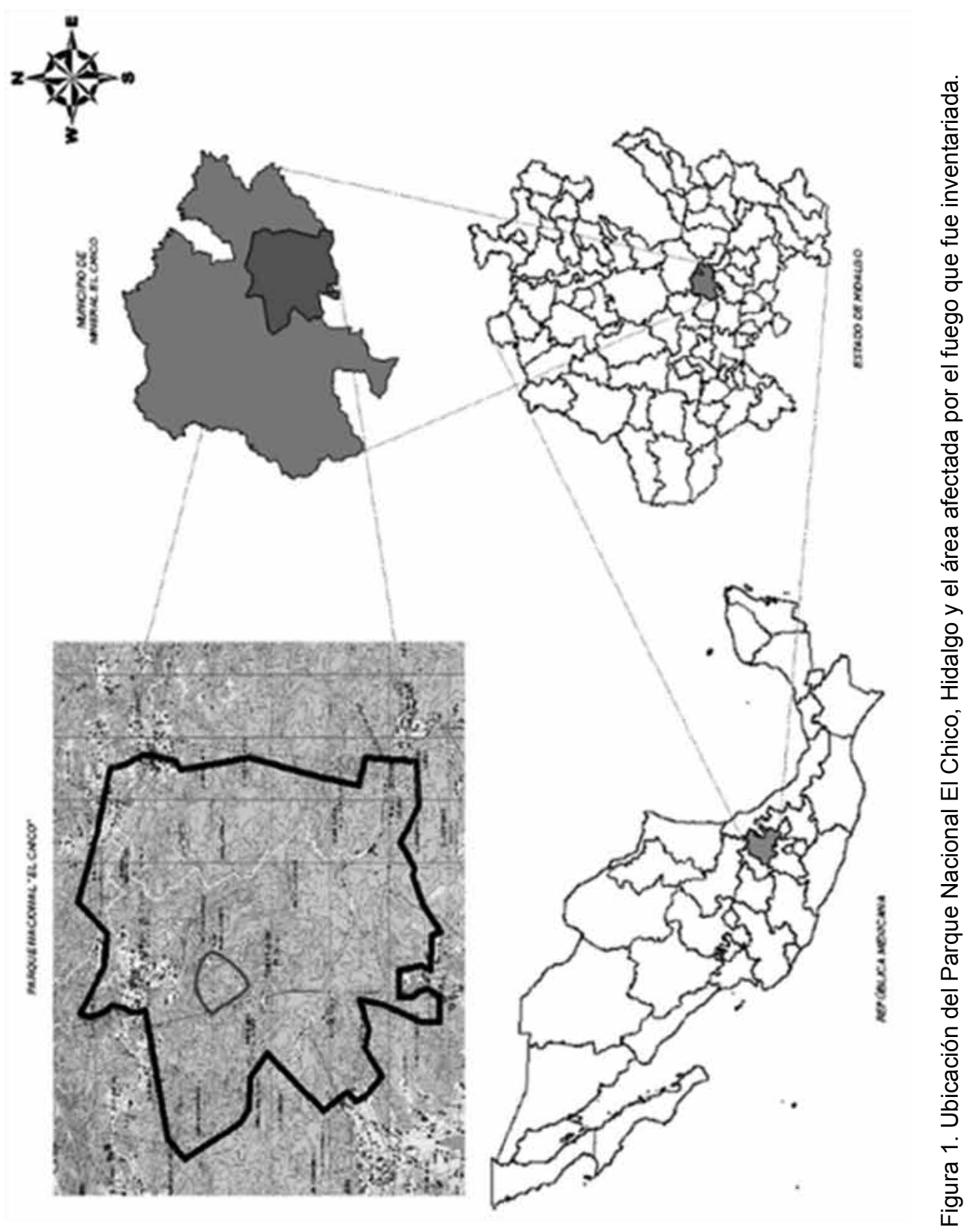


Dado que el reglamento de las Áreas Naturales Protegidas y el Programa de Manejo del Parque Nacional El Chico no permiten el uso de métodos destructivos en la vegetación (Conanp, 2005), la biomasa y carbono existentes se estimaron con base en un inventario, mediante el uso de ecuaciones matemáticas que permitieron determinar las variables dependientes a partir de la medición del diámetro normal y altura total de los árboles. El diámetro mínimo que se tomó en cuenta para el arbolado y regeneración natural fue de $5,0 \mathrm{~cm}$ y $2,5 \mathrm{~cm}$, respectivamente. La información dasométrica para el estudio provino de 15 sitios circulares de muestreo de $1000 \mathrm{~m}^{2}$ (lo que equivale a $5 \%$ de la zona donde ocurrió el incendio), distribuidos de manera sistemática en la superficie afectada por el incendio forestal. La base de datos la constituyeron 112 fustes de árboles muertos, 28 árboles adultos vivos y 4515 renuevos de oyamel que se evaluaron en los sitios muestreados. Para obtener la biomasa $(Y)$ de cada árbol se consideró el valor de $360 \mathrm{~kg} / \mathrm{m}^{3}$ que corresponde a la densidad de la madera de Abies religiosa (D), determinada por Goche et al. (2000). Con los datos tomados en campo: diámetro medido a $1,30 \mathrm{~m}$ del suelo y altura total para cada fuste $(H)$, se procedió a realizar el cálculo del área basal $(A B)$ y la biomasa por individuo, tomando como base la siguiente ecuación desarrollada por el grupo Fundación Solar (2000) para biomasa en árboles muertos en pie, vivos y renuevos:

$$
Y=A B^{\star} H^{\star} D
$$

Considerando que los fustes no son cilíndricos, se agregó a la ecuación anterior el coeficiente de forma por categorías de diámetro y altura para oyamel, publicados por la SARH (1985), resultando la ecuación siguiente:

$$
Y=A B^{\star} H^{\star} C f^{\star} D
$$

donde:

$$
\begin{array}{ll}
Y= & \text { biomasa (kilogramos) } \\
A B= & \text { área basal (metros cuadrados) } \\
H= & \text { altura total del árbol (metros) } \\
C f= & \text { coeficiente de forma para oyamel } \\
D= & \text { densidad de la madera de oyamel } \\
& \left(\mathrm{kg} / \mathrm{m}^{3}\right)
\end{array}
$$

Para determinar el coeficiente de carbono se tomaron muestras de madera del interior del fuste en 5 árboles seleccionados al azar, a la altura de 1,30 m. Las muestras se secaron en una estufa a una temperatura de $105^{\circ} \mathrm{C}$ hasta alcanzar peso constante, posteriormente se pulverizaron en un molino tipo mortero y se determinó su contenido de carbono total mediante el equipo Solids TOC Analyzer ${ }^{\circledast}$. Se obtuvo un valor promedio del coeficiente de carbono, este valor se multiplicó por la biomasa individual de los árboles para obtener la cantidad de carbono.

Con los datos estimados de biomasa y carbono individual y el correspondiente diámetro normal de los árboles muestreados, se ajustó el modelo matemático expresado en su forma potencial $Y=b_{0} D N^{b}{ }_{1} \mathrm{y}$ logarítmica $\ln (Y)=\ln \left(b_{0}\right)+b_{1} \ln (D N)$. Los modelos fueron ajustados por mínimos cuadrados con el propósito de obtener los valores de los parámetros $b_{0}$ y $b_{1}$ que representan la ordenada al origen y la pendiente del modelo de regresión, respectivamente. Se utilizó el paquete estadístico (Statistica ver 6.0), buscando que los modelos presentaran un buen ajuste en los criterios de bondad ( $R^{2}$, valor de $F$ ), además de que fueran de fácil aplicación práctica. Finalmente, se construyó una tabla con los resultados de este estudio y los obtenidos aplicando las ecuaciones desarrolladas por Avendaño et al. (2009) para la misma especie en bosques del estado de Tlaxcala, para comparar la biomasa y el carbono residual con base en el diámetro normal (DN) en los árboles muertos, vivos y renuevo de oyamel. 


\section{RESULTADOS}

\section{Estructura dasométrica actual del bosque}

Arbolado muerto en pie. En el área afectada por el incendio forestal, el mayor número de árboles adultos muertos que permanecen en pie y que fueron inventariados corresponden a las categorías diamétricas de $15 \mathrm{~cm}, 30 \mathrm{~cm}$ y $35 \mathrm{~cm}$, con un número de individuos por hectárea de 9 , 9 y 11 respectivamente (el diámetro mínimo encontrado fue de $11,4 \mathrm{~cm}$ y el máximo de $88,9 \mathrm{~cm}$ ) y con presencia de árboles adultos muertos en prácticamente todas las categorías diamétricas en un intervalo de $10 \mathrm{~cm}$ a $90 \mathrm{~cm}$ (Fig. 2), lo cual muestra que la condición original del bosque de oyamel (antes del incendio) correspondía a un tipo de masa forestal irregular característica de esta especie tolerante, que presentaba individuos de diferentes categorías diamétricas.

Árboles vivos residuales. El mayor número de árboles adultos inventariados que lograron sobrevivir al siniestro muestran categorías diamétricas de $35 \mathrm{~cm}, 45$ $\mathrm{cm}$ y $60 \mathrm{~cm}$ con un número de individuos por hectárea de 4, 3 y 2, respectivamente (Fig. 3). En su mayoría son árboles maduros dispersos que después de ocurrido el incendio siguieron produciendo semilla para el establecimiento de la regeneración natural de oyamel en el área afectada.

Regeneración natural de oyamel. Después de ocurrido el incendio forestal, en las áreas afectadas se observó un gran número de especies arbustivas y herbáceas que sirvieron de nodrizas, proporcionando la sombra que requiere el oyamel para su establecimiento. A 12 años de

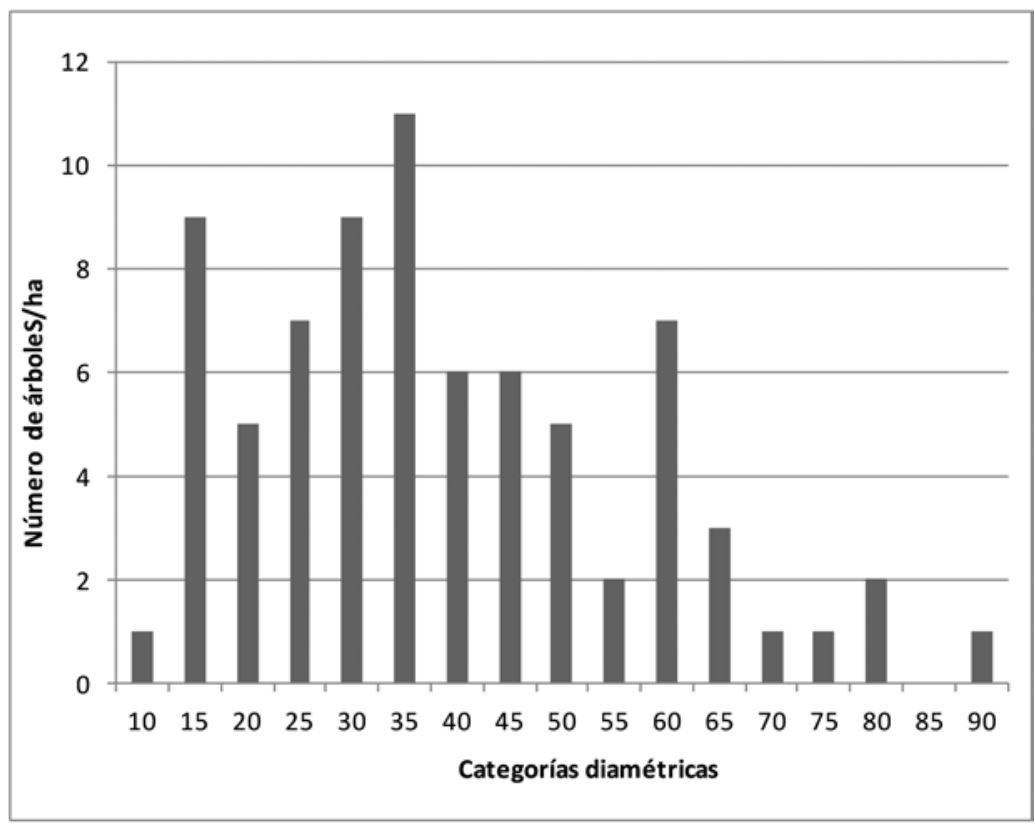

Figura 2. Número de árboles muertos de Abies religiosa, por hectárea, y sus categorías diamétricas. 


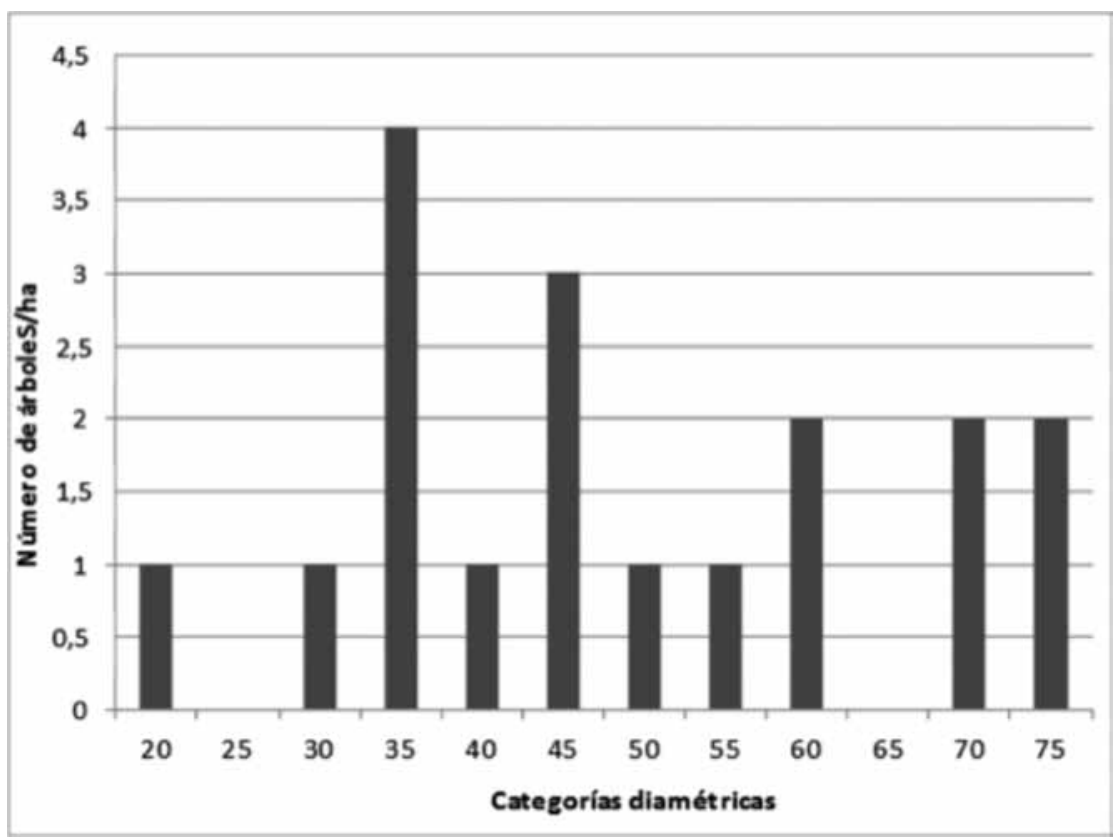

Figura 3. Número de árboles adultos de Abies religiosa, por hectárea, que lograron sobrevivir después de ocurrido el incendio.

ocurrido el siniestro se han establecido en el sitio 3010 árboles por hectárea, los árboles que se encontraron durante el inventario correspondieron a las categorías de $5 \mathrm{~cm}$ y $10 \mathrm{~cm}$ con 2000 y 1010 brinzales, respectivamente (Fig. 4).

\section{Estimación de biomasa}

La biomasa estimada en los árboles muertos, arbolado vivo y renuevo de oyamel, se realizó con el modelo en su forma potencial, mostrando un buen ajuste con un coeficiente de determinación de $R^{2}=0,99$. Este valor significa la confiabilidad con la que un modelo puede generar resultados adecuados, siendo más aceptable a medida que se acerque a uno (Rodríguez et al., 2006). En la figura $5 a$ se observa de manera gráfica la tendencia ascendente en la biomasa de acuerdo con las categorías diamétricas de los árboles objeto de estudio, y al hacer lineal el modelo (Fig. $5 b)$ se facilita la interpretación del análisis de regresión, tal como lo recomiendan Little y Jackson (1976).

Resultados similares fueron encontrados por Pimienta et al. (2007) en un estudio para determinar biomasa en arbolado vivo de Pinus cooperi, en Pueblo Nuevo, Durango, con un modelo que considera las variables dasométricas de diámetro y altura. Otros estudios han utilizado la misma ecuación, obteniendo resultados satisfactorios: Acosta et al. (2002) presentó una $\mathrm{R}^{2}$ de 0,97 para biomasa aérea en especies forestales nativas del bosque mesófilo de montaña en la Sierra Norte de Oaxaca. Avendaño et al. (2009), empleando este mismo modelo, encontraron un $\mathrm{R}^{2}$ de 0,99 para Abies religiosa en un bosque del estado de Tlaxcala. 


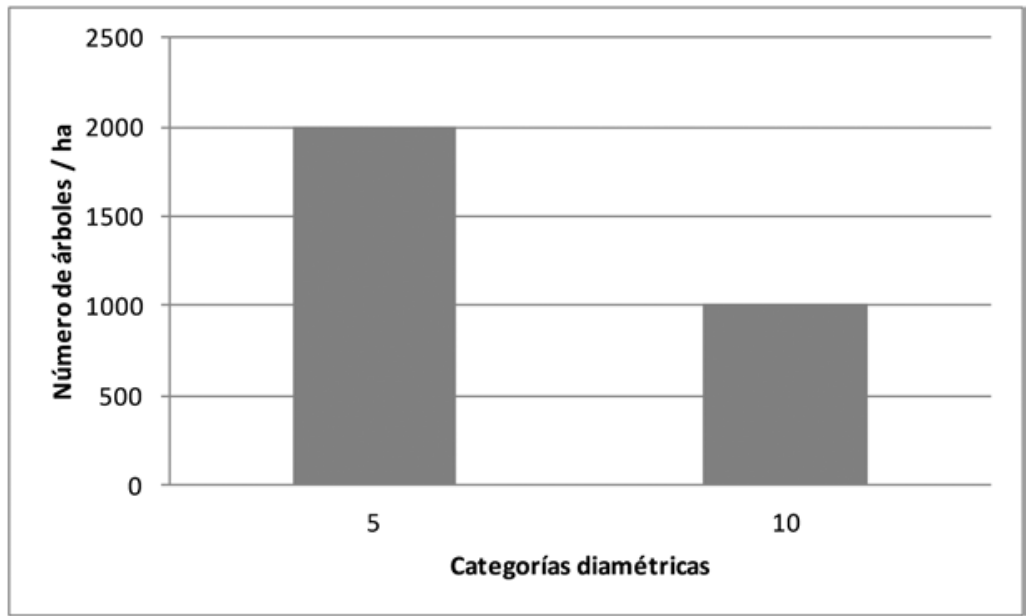

Figura 4. Número de árboles de Abies religiosa, por hectárea, que se establecieron en forma natural después de ocurrido el incendio.
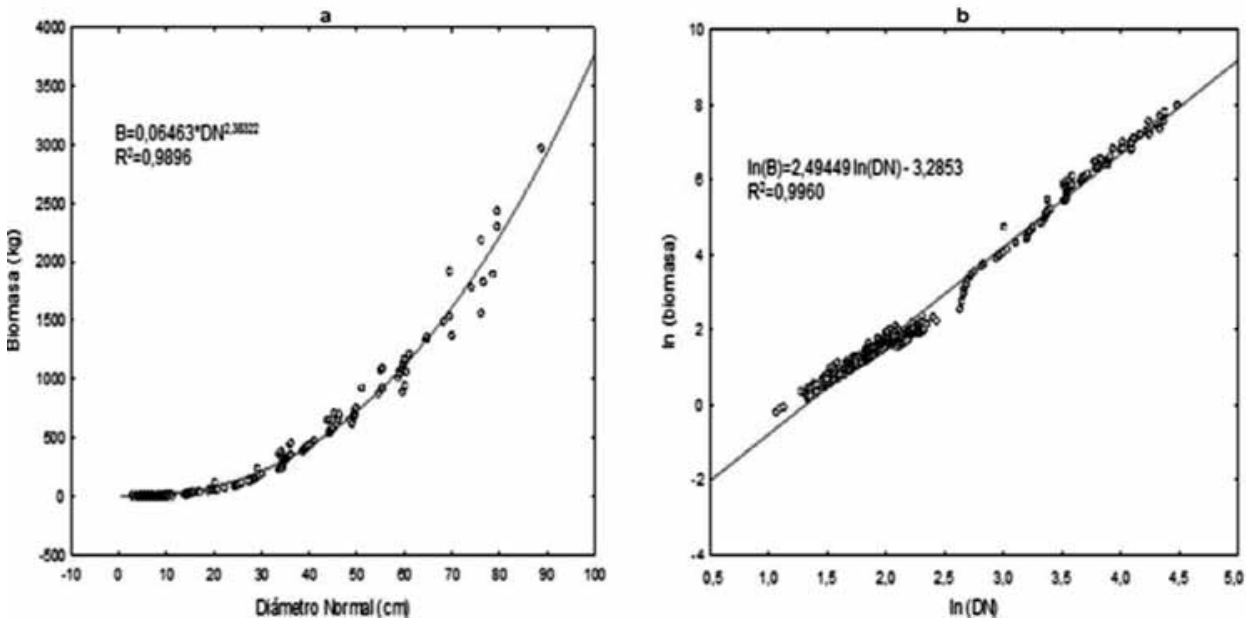

Figura 5. a. Modelo Potencial (a) y convertido a lineal (b) para estimar biomasa en función del DN para árboles de oyamel del Parque Nacional El Chico. 


\section{Estimación de carbono}

Después de determinar la biomasa para cada árbol, el valor obtenido se multiplicó por la concentración de carbono que se obtuvo para el oyamel $(0,45)$, valor muy cercano al utilizado por Acosta et al. (2009) que fue de 0,46. Posteriormente, se generó el modelo Potencial $C=0.029083^{*} D N^{2,38322}$, con un coeficiente de determinación $\left(R^{2}=0,99\right)$, satisfactorio para estimar carbono en árboles residuales y establecidos después de un incendio forestal de tipo superficial (Fig. 6).

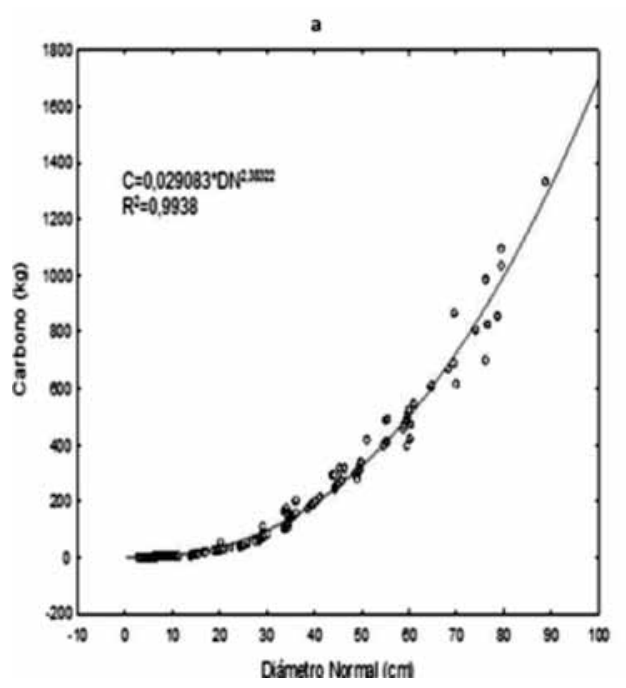

Mediante los modelos generados para estimar biomasa y carbono, se elaboró la tabla 1 que muestra los valores individuales y por hectárea, considerando como única variable el diámetro normal, que facilita la estimación de manera rápida y confiable de la biomasa y el carbono almacenado en el bosque de oyamel. Los resultados de este estudio se compararon con los valores obtenidos por los modelos generados por Avendaño et al. (2009) para un bosque de oyamel en el estado de Tlaxcala.

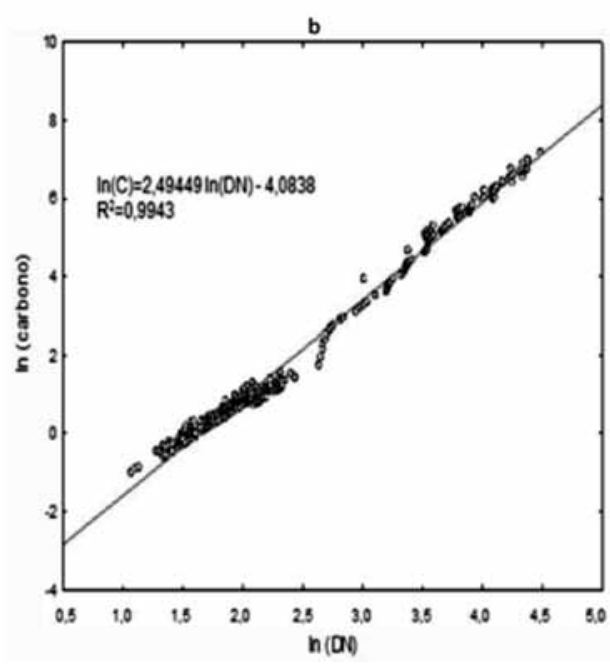

Figura 6. Carbono en función del DN para árboles muertos en pie, arbolado adulto vivo y renuevo establecido en forma natural en un área afectada por un incendio forestal.

Los valores de biomasa, carbono individual y carbono por hectárea obtenidos con los modelos generados en este estudio, y los de Avendaño et al. (2009) en las categorías diamétricas inferiores $(5 \mathrm{~cm}, 10 \mathrm{~cm}$ y $15 \mathrm{~cm})$ son muy cercanos. Para el resto de las categorías, las estimaciones con los modelos generados para el bosque de oyamel del Parque Nacional El Chico son más conservadoras.
Después de ocurrido el incendio forestal que afectó 30,34 ha en el Parque Nacional El Chico, se tiene una biomasa producto de los árboles muertos en pie de 41,20 t/ha, que representan 18,54 $\mathrm{t}$ de carbono por hectárea. Los árboles adultos que lograron sobrevivir al siniestro contienen una biomasa de $7,51 \mathrm{t} /$ ha que equivalen a 3,38 t/ha de carbono que no fueron liberadas hacia la atmósfera (Tabla 2). 


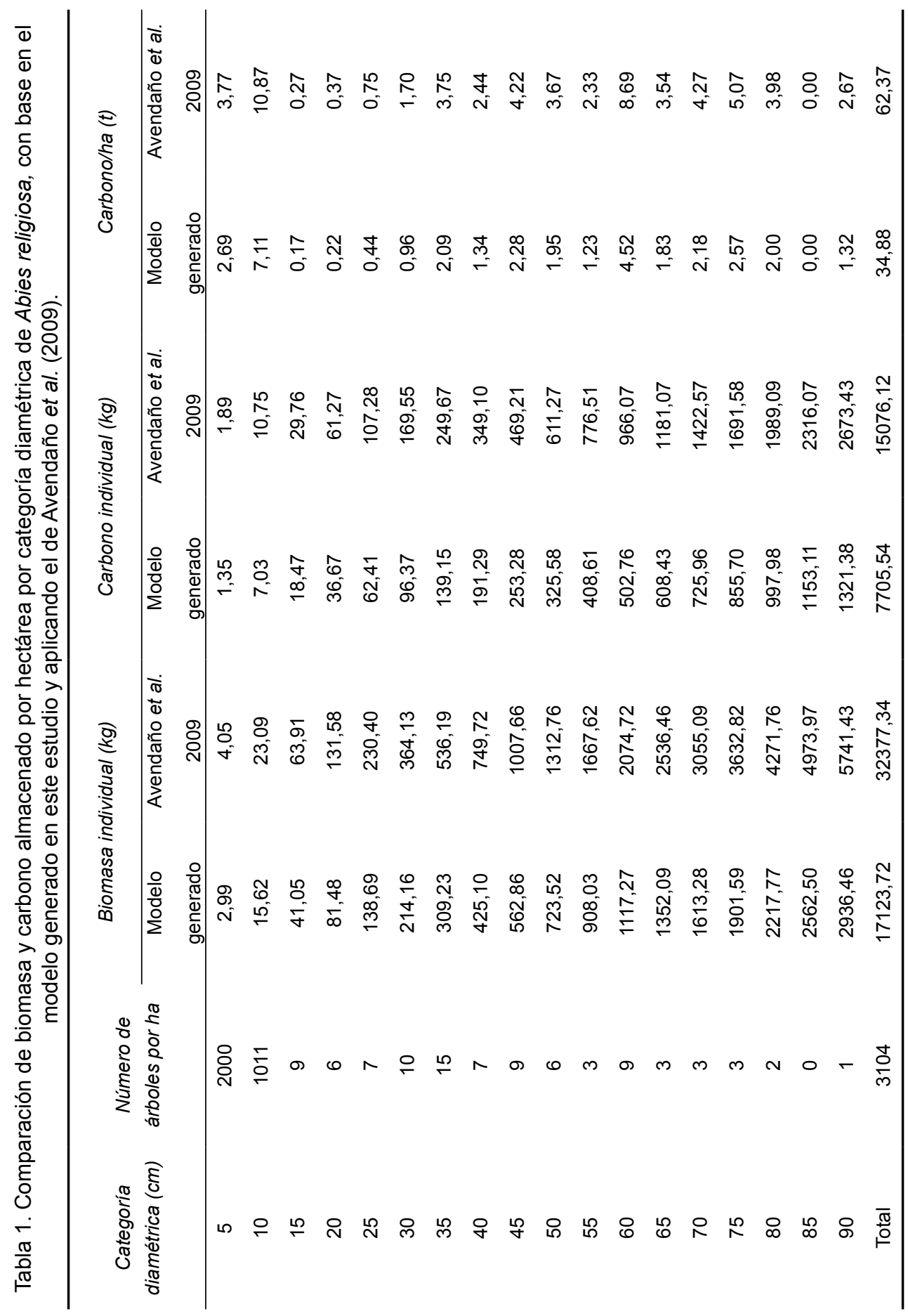


Tabla 2. Estimación de carbono por hectárea para cada condición del arbolado, utilizando el modelo generado para el bosque de oyamel del Parque Nacional El Chico.

\begin{tabular}{cccc}
\hline $\begin{array}{c}\text { Condición del } \\
\text { arbolado }\end{array}$ & $\begin{array}{c}\text { Rango de categorías } \\
\text { diamétricas }\end{array}$ & Número de árboles/ha & $\begin{array}{c}\text { Carbono/ha } \\
(t)\end{array}$ \\
\hline Renuevo & 5 a 10 & 3010 & 9,8 \\
Arbolado vivo & 20 a 75 & 18 & 3.38 \\
Arbolado muerto & 10 a 90 & 76 & 18,54 \\
\hline
\end{tabular}

Asimismo, a 12 años de ocurrido el incendio forestal, el renuevo de oyamel ha almacenado 9,8 t/ha de carbono, esto es $297,3 \mathrm{t}$ de carbono en las 30,34 ha siniestradas. La eficiencia en el secuestro se podría incrementar mediante la aplicación de tratamientos silvícolas (FAO, 2010), tales como las cortas de aclareo que en forma gradual favorecen el crecimiento en diámetro, volumen y biomasa de los árboles.

\section{DISCUSIÓN}

Considerando la opinión de algunos autores como Avendaño et al. (2009), respecto a que en un árbol completo $84,5 \%$ de la biomasa se almacena en el fuste, $6,9 \%$ en las ramas y $8,6 \%$ en el follaje, con los datos obtenidos en este trabajo sobre la estimación del contenido de carbono en árboles muertos y vivos del área afectada por el incendio, se pudo conocer la capacidad de regeneración y el potencial de este bosque para el almacenamiento de carbono. También estos datos son importantes para complementar las evaluaciones e informes de daños causados por los incendios forestales, tomando en cuenta que cuando ocurre un incendio forestal de tipo superficial no todo el carbono regresa a la atmósfera. Muchos árboles que no fueron consumidos por el fuego permanecen en el sitio por mucho tiempo, ya que la muerte natural o el biodeterioro requiere desde varias semanas hasta muchas décadas para completar la descomposición de la biomasa (dependiendo de la condiciones del sitio), dejando parte en el suelo y la otra directamente en la atmósfera. La cosecha forestal puede almacenar parte del carbono vegetativo por largos periodos como madera sólida en productos de larga duración (construcción en madera). Los desechos de transformación y las especies no comerciales se dejan para el deterioro o combustible, por lo que se recomienda dejarlos en pie o acomodarlos en obras de control de erosión del suelo, haciendo posible que un alto porcentaje de carbono se mantenga por más tiempo en el sitio y que después se incorpore de forma gradual al suelo (FAO, 2010). Con acciones como éstas, y el establecimiento de la regeneración natural en los sitios afectados por el fuego se reactiva el ciclo del carbono en este ecosistema, lo cual contribuye a mitigar el cambio climático. El contenido de biomasa y carbono que quedó en el ecosistema después del incendio puede servir para estimar la línea base de proyectos de carbono en ese tipo de ecosistemas.

Los modelos generados en este estudio, de preferencia deben ser aplicados en ecosistemas similares de la sierra de Pachuca, donde no sea factible la 
cuantificación de biomasa con métodos destructivos. Como mencionan Dávalos et al. (2008), las técnicas de estimación de biomasa en general son muy costosas, debido al enorme volumen de material que se requiere colectar para generar las ecuaciones de cálculo, por lo que se puede considerar para esa estimación la densidad de la madera de las especies de interés, junto con la información de las dimensiones de los árboles y muestreos de la biomasa total de la vegetación leñosa.

Los valores obtenidos mediante la aplicación de los modelos generados en este estudio y los desarrollados por Avendaño et al. (2009) para el carbono individual, son mayores con los modelos generados por estos últimos autores (Fig. 7). Lo cual puede ser resultado de que en este estudio se utilizó de manera generalizada el valor de la densidad de la madera para estimar la biomasa de los árboles individuales completos, mientras que en el estudio realizado por Avendaño et al. (2009) se recurrió al uso del método destructivo para la obtención de la biomasa en los diferentes componentes del árbol, lo cual es más preciso.

Aun así, el uso de los modelos generados en este estudio permite la estimación de biomasa y carbono en los bosques de oyamel que se desarrollan en una zona de gran afluencia turística como es el Parque Nacional El Chico, donde no es recomendable el derribo de arbolado para la cuantificación de estos elementos, por el gran impacto visual y social que se genera.

\section{CONCLUSIONES}

Las ecuaciones generadas para estimar la biomasa y carbono en árboles de Abies religiosa en un área afectada por un incendio forestal fueron $B=0,06463^{*} D N^{2,38322}$ y

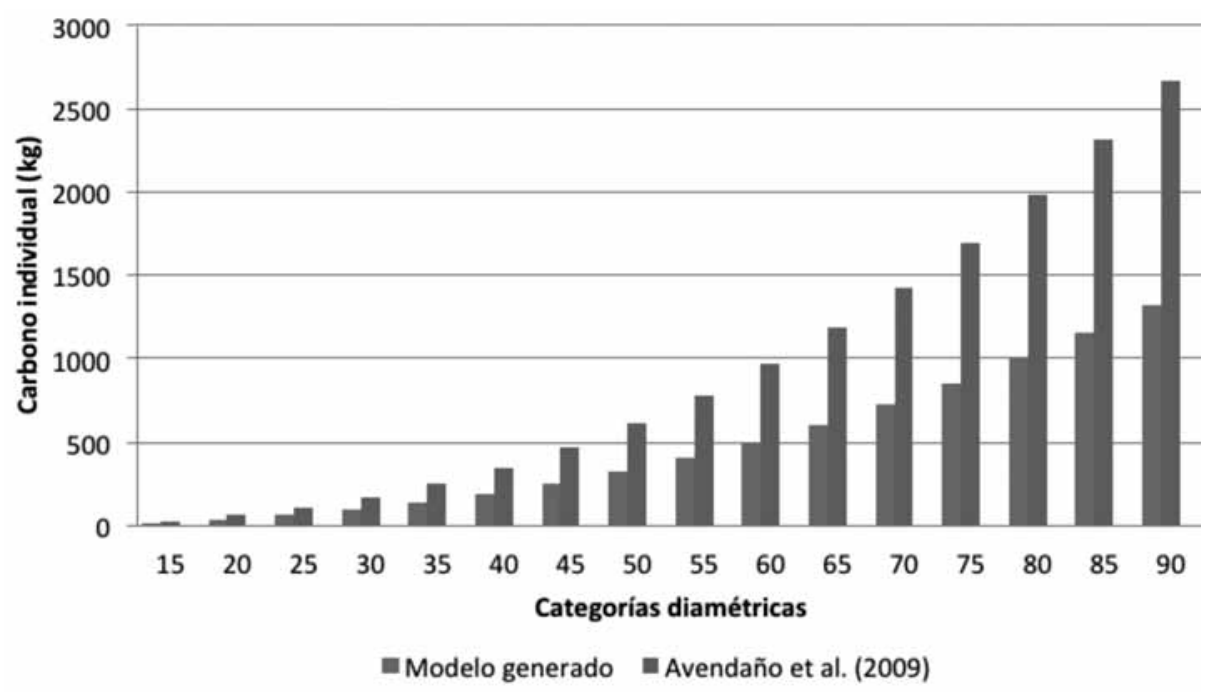

Figura 7. Valores de carbono individual obtenidos con el modelo generado en este estudio y el desarrollado por Avendaño et al. (2009), para el bosque de oyamel del Parque Nacional El Chico. 
$C=0,029083^{*} D N^{2,38322}$, respectivamente, mostraron un buen ajuste, por lo que son adecuadas y confiables para usarse en áreas siniestradas con condiciones similares, para la misma especie y región, como en Áreas Naturales Protegidas donde no es posible utilizar métodos destructivos para el cálculo de biomasa y carbono.

En las 30,34 hectáreas siniestradas por el incendio forestal ocurrido en 1998 en el Parque Nacional El Chico, un total de 665,05 t de carbono no se consumieron por el fuego, es decir, no fueron liberadas hacia la atmósfera. Se suma una captura actual de 297,33 t de carbono por el renuevo establecido a 12 años de ocurrido el siniestro.

Para aumentar la capacidad de almacenamiento de carbono se recomienda la aplicación de cortas de aclareo en las áreas con renuevo establecido, con el fin de favorecer el aumento de biomasa en los árboles residuales y prevenir posibles daños por la incidencia de incendios forestales.

\section{REFERENCIAS}

Acosta M., M, F. Carrillo A. y M. Díaz L. 2009. Determinación del carbono total en bosques mixtos de Pinus patula Schl. et Cham. Terra Latinoamericana 27(2):105-114.

Acosta M., M., J. Vargas H., J.D. Etchevers B. y A. Velázquez M. 2002. Estimación de la biomasa aérea mediante el uso de relaciones alométricas en seis especies arbóreas en Oaxaca, México. Agrociencia 6:725736.

Amezcua C., P. y H.L. Valderrama L. 1999. Productividad de suelos con Abies religiosa Schl. et Cham. en el Parque Nacional "El Chico", Hidalgo. Tesis de Licenciatura. Departamento de Suelos. Universidad Autónoma Chapingo.121p.

Avendaño H., D.M., M. Acosta M., F. CarriIlo A. y J.D. Etchevers B. 2009. Estimación de biomasa y carbono en un bosque de Abies religiosa. Fitotecnia Mexicana 32(3):233-238.

Cadena M., O.I. y G. Ángeles P. 2005. Almacenes de carbono en hojarasca en bosques manejados de Pinus patula en Zacualtipán, Hidalgo. Resumen del VII Congreso Mexicano de Recursos Forestales. Chihuahua, Mex. p:422-423.

Conanp (Comisión Nacional de Áreas Naturales Protegidas). 2005. Programa de conservación y manejo del Parque Nacional El Chico. México. 236 p.

Cuevas G., R., E.A. Cisneros L., E.J. Jardel P., E.V. Sánchez R., L. Guzmán H., N. M. Núñez L. y C. Rodríguez G. 2011. Análisis estructural y de diversidad en los bosques de Abies de Jalisco, México. Revista Mexicana de Biodiversidad 82:1219-1233.

Dávalos S., R., M.I. Morato y E. Martínez P.C. 2008. Almacenamiento de carbono. In: R.H.Manson, V. Hernández-Ortiz, S. Gallina y K. Mehltreter, Eds. Agroecosistemas cafetaleros de Veracruz: Biodiversidad, manejo y conservación. Inecol, INE-Semarnat. México, D.F. p:223-233.

FAO (Organización de Naciones Unidas para la Alimentación y la Agricultura). 2010. La gestión de los bosques ante el cambio climático. 20 p.

Fundación Solar. 2000. Elementos técnicos para inventarios de carbono. Guatemala. $31 \mathrm{p}$. 
Fulé, P.Z. y W.W. Covington. 1997. Fire regimes and forest structure in the Sierra Madre Occidental, Durango, México. Acta Botánica Mexicana 41:43-79.

García, E. 1981. Modificaciones al sistema de clasificación climática de Köppen. Instituto de Geografía UNAM. México.166p.

Goche T., J.R., M. Fuentes S., A. Borja D. y H. Ramírez M. 2000. Variación de las propiedades físicas de la madera en un árbol de Abies religiosa y de Pinus ayacahuite var. veitchii. Revista Chapingo: Serie Ciencias Forestales y del Ambiente 6(1):83-92

Ikkonen, E., E. Ángeles C. y N.E. García C. 2004. Producción de $\mathrm{CO}_{2}$ en andosoles afectados por incendios forestales en el Parque Nacional El Chico, Hidalgo. Terra Latinoamericana. 22(4):425-431

IPCC (Panel Intergubernamental sobre el Cambio Climático). 2000. Land use, land-use change, and forestry special report. Cambridge University Press. Cambridge. 377 p.

Little, T.M. y F. Jackson. 1976. Métodos estadísticos para la investigación en la agricultura. Trillas. México. 270 p.

Ordóñez, B., H.J. De Jong y O. Masera. 2001. Almacenamiento de carbono en un bosque de Pinus pseudostrobus, Michoacán. Madera y Bosques 7(2):27-47.

Pimienta D., D.J., G. Domínguez C., O. Aguirre C., F.J. Hernández y J. Jiménez P. 2007. Estimación de biomasa y contenido de carbono de Pinus cooperi Blanco, en Pueblo Nuevo, Durango. Madera y Bosques 13(1):35-46.

Rodríguez L., R., J. Jiménez P., O. Aguirre C. y E.J. Treviño G. 2006. Estimación del carbono almacenado en un bosque de niebla en Tamaulipas, México. Ciencia UANL 9(2):179-187

SARH. 1985. Memoria del Inventario Forestal del estado de Hidalgo, Manejo y aprovechamiento de los recursos forestales. $69 \mathrm{p}$.

Schroeder, P., S. Brown, J. Mo, R. Birdsey y C. Cieszewwski. 1997. Biomas estimation for temperate broadleaf forests of the United States using inventory data. Forest Science. 43(3):424-434.

Tipper R. 1998. Update on carbon offsets. Tropical Forest Update 8(1):2-5.

Torres, R., J.M. y A. Guevara S. 2002. El potencial de México para la producción de servicios ambientales: captura de carbono y desempeño hidráulico. Gaceta Ecológica INE n. 63. 2013. Estimación de biomasa y carbono almacenado en árboles de oyamel afectados por el fuego en el Parque Nacional "El Chico", Hidalgo, México. Madera y Bosques 19(2):73-86. 